UCRL 5648

\title{
UNIVERSITY
CALIFORNIA
}

\section{Ernest O. Laurence}
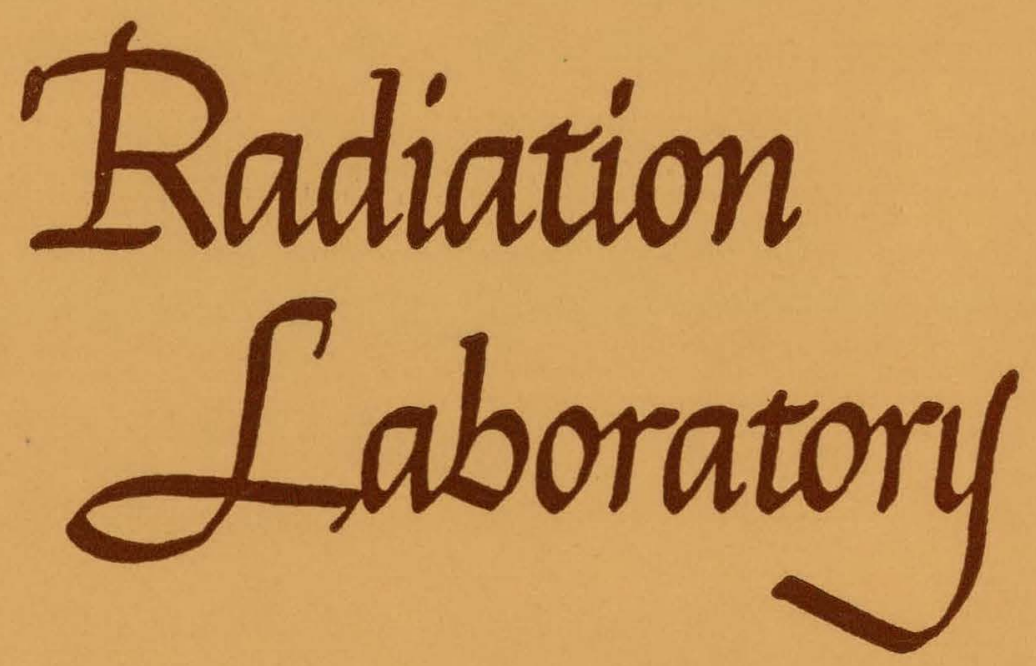


\section{DISCLAIMER}

This report was prepared as an account of work sponsored by an agency of the United States Government. Neither the United States Government nor any agency Thereof, nor any of their employees, makes any warranty, express or implied, or assumes any legal liability or responsibility for the accuracy, completeness, or usefulness of any information, apparatus, product, or process disclosed, or represents that its use would not infringe privately owned rights. Reference herein to any specific commercial product, process, or service by trade name, trademark, manufacturer, or otherwise does not necessarily constitute or imply its endorsement, recommendation, or favoring by the United States Government or any agency thereof. The views and opinions of authors expressed herein do not necessarily state or reflect those of the United States Government or any agency thereof. 


\section{DISCLAIMER}

Portions of this document may be illegible in electronic image products. Images are produced from the best available original document. 


\section{UNIVERSITY OF CALIFORNIA}

Lawrence Radiation Laboratory

Livermore, California

Contract No. W-7405-eng-48

AIR-CORE STRONG FOCUSING SYNCHROTRON

Nicholas C. Christofilós

August 21, 1959

Printed for the U.S. Atomic Energy Commission 


\title{
AIR-CORE STRONG FOCUSING SYNCHROTRON *
}

\author{
Nicholas C. Christofilos \\ Lawrence Radiation Laboratory, University of California \\ Livermore, California
}

\begin{abstract}
An air-core strong focusing synchrotron is described. Low temperature magnets, $80^{\circ} \mathrm{K}$ or lower are proposed. High magnetic field value and high acceleration rate are characteristic advantages of the air-core magnets. The machine becomes smaller while the required radiofrequency power is considerably increased. Present accelerators can be classified in either of two extremes:

a. Closed orbit accelerators where one can hardly see the accelerating element; the main component is the guiding magnet and the acceleration time is of the order of a second.

b. Linear accelerators where the particles travel once through the machine and, due to this fact, tremendous amounts of $r f$ power are required. The proposed machine is a compromise between these extremes; hence, the cost of the magnet and accelerating equipment is well balanced; the acceleration time is of the order of a few tens of milliseconds.

Molecular beam injection is proposed, allowing multi-turn injection; thus trapping of $6 \times 10^{12}$ particles per pulse is possible. By employing high magnetic fields it is possible to reduce the aperture; hence, the final beam cross-sectional area becomes very small, allowing synchroçlash operation of two machines without the necessity of storage, with satisfactory reaction yield. The magnet units are combined with the radiofrequency cavities. This arrangement eliminates long straight sections except at the injection and targets.
\end{abstract}

Parameters of a 24-Bev machine of this type are cited. A method of avoiding blow-up of the beam at the phase transition is described. By properly changing the amplitude of the rf voltage near the phase transition energy, it is possible to obtain solutions of constant amplitude for both the momentum and phase oscillations.

* This work was performed under the auspices of the U.S. Atomic Energy Commission. 


\title{
AIR-CORE STRONG FOCUSING SYNCHROTRON
}

\author{
Nicholas C. Christofilos \\ Lawrence Radiation Laboratory, University of California \\ Livermore, California
}

\section{INTRODUCTION}

There are two distinct directions to be considered in the planning of future high-energy accelerators: High energy, much higher than the accelerators presently under construction (CERN, Switzerland; Brookhaven National Laboratory, U.S.A.), namely of the order of $100 \mathrm{Bev}$; or moderate energy, 10 to $30 \mathrm{Bev}$, but much higher current of the order of a few microamperes.

Steel magnets have certain inherent limitations. In the case of very high energy, the circumference of a steel magnet becomes undesirably large. The only way to reduce the length of the circumference is to use higher values of magnetic field (i.e., 50,000 gauss) which are possible only with air-core magnets. The air-core magnets are usually associated with high stored energy. However, with proper coil design it is possible to reduce the stored energy to the same value as in a steel-magnet machine for the same particle energy. This can be obtained by reducing the size-of the magnet, and by proper arrangement of the current distribution in the coils. The peak power requirements of such a magnet are rather high. However, this can be considerably reduced by operating the magnet at a temperature of $80^{\circ} \mathrm{K}$ or lower. Recent development in cryogenics, or rather the necessity of building large refrigeration units for other applications, resulted in a considerable reduction of the cost of such units. Hence, application of cryogenics in accelerators is now under consideration, although positive conclusions are not yet possible. In addition to the reduction of the required peak power it is possible to reduce considerably the average power by contemplating a short acceleration time, less than one-tenth of a second. This shorter acceleration time requires in turn a more powerful rf amplifier but this is necessary anyway, if it is de sired to accelerate a large number of particles per pulse, to compensate for azimuthal space charge forces in the bunch. An additional advantage of the high field value is that the particle density in the bunch becomes high enough so that synchroclash operation might be possible without the necessity of storage rings. 


\section{MAGNET COILS}

The magnet coils are confined between two coaxial cylinders of radius $\mathbf{r}_{i}$ and $\mathbf{r}_{0}$ respectively (Fig. 1). The cylinder of radius $r_{i}$ is the vacuum chamber. Thus the aperture of the machine is of circular cross section. The required field in the aperture is a dipole field (the guiding field) combined with a quadrupole focusing field. The solution of the vector potential within the coil must satisfy the boundary conditions; namely, to match the field components of the vacuum field in the aperture and in the space outside the coil. The current distribution $(j)$ within the coil is then determined from the equation

$$
\frac{4 \pi j}{10}=-\nabla \times\left(\nabla \times A_{z}\right)
$$

where $z$ is along the axis of the coil.

There is a variety of functions which can satisfy these conditions. However, the calculations are much simplified by. employing Bessel functions as solutions for the vector potential within the coil. Hence, the solutions can be identical with solutions of Maxwell equations for time-varying fields where the displacement current has been substituted by the actual current distribution in the coil. These solutions for each harmonic (of $\underline{\text { nth }}$ order) assume the form

$$
A_{z}=-\frac{B_{0}}{k} C_{n}(k r) \cdot \cos (n \theta)
$$

where

$$
C_{n}(k r)=c_{1} J_{n}(k r)+c_{2} Y_{n}(k r)
$$

and $J_{n}, Y_{n}$ are the Bessel functions of $\underline{n}$ th order of the first and second kind, respectively.

The boundary conditions are (see Appendix I)

$$
\begin{aligned}
C_{n+1}\left(k r_{i}\right) & =0 \\
C_{(n-1)}\left(k r_{0}\right) & =0 .
\end{aligned}
$$

By integrating the square of the field and the current density we can determine the stored energy and the power loss respectively, as a function of the ratio $r_{0} / r_{i}$ of the outer to the inner radius of the coil. A plot of these two quantities as a function of $r_{0} / r_{i}$ is shown in Fig. 2. 'l he stored energy varies almost linearly with $r_{0} / r_{i}$ while the power loss'increases rapidly for values of: $r_{0} / r_{i}$ less than.2. In the example cited hereafter a ratio of $r_{0} / r_{i} \approx 3$ 
has been selected. For this particular example, the current density distribution $j$, the power loss $W_{R}$, and the stored energy $W_{0}$ are given by the following equations:

$$
j=\frac{2}{\pi} \frac{B_{0}}{r_{i}}\left[C_{1}\left(k_{1} r\right) \cos \theta+1.5775 a C_{2}\left(k_{2} r\right) \cos 2 \theta\right] a m p s / c^{2},
$$

where

$$
\begin{aligned}
\mathrm{C}_{1}\left(\mathrm{k}_{1} \mathrm{r}\right)= & 2.3711 \mathrm{~J}_{1}\left(\mathrm{k}_{1} \mathrm{r}\right)+0.076221 \mathrm{Y}_{1}\left(\mathrm{k}_{1} \mathrm{r}\right) \\
\mathrm{C}_{2}\left(\mathrm{k}_{2} \mathrm{r}\right)= & 3.9448 \mathrm{~J}_{2}\left(\mathrm{k}_{2} \mathrm{r}\right)+0.04738 \mathrm{Y}_{2}\left(\mathrm{k}_{2} \mathrm{r}\right) \\
\mathrm{J}_{1} \text { and } \mathrm{J}_{2} \text { are } & \text { Bessel functions of the first kind of the first and } \\
& \text { second order, respectively. } \\
\mathrm{Y}_{1} \text { and } \mathrm{Y}_{2} \text { are } & \text { Bessel functions of the second kind of the first and } \\
& \text { second order, respectively. } \\
\mathrm{k}_{1} \mathrm{r}_{\mathrm{i}}= & 0.8 \\
\mathrm{k}_{2}= & 1.5775 \mathrm{k}_{1} \\
\mathrm{~B}_{0}= & \text { peak value of the field at the orbit (gauss) } \\
\mathrm{r}_{\mathrm{i}}= & \text { the inner radius of the coil in cm } \\
\mathrm{a}= & \text { the ratio of the quadrupole to the dipole field strength } \\
& \text { at } \mathrm{r}=\mathrm{r}_{\mathrm{i}} \text {. }
\end{aligned}
$$

The power loss in the coil is:

$$
\begin{gathered}
\mathrm{w}_{\mathrm{r}}=(\rho / \eta) \mathrm{B}_{0}^{2} \mathrm{k}_{\mathrm{i}}^{2} \mathrm{r}_{\mathrm{i}}^{2} \\
\left\{\left(\mathrm{r}_{0}^{2} / \mathrm{r}_{\mathrm{i}}{ }^{2}\right) \mathrm{C}_{1}{ }^{2}\left(\mathrm{k}_{1} \mathrm{r}_{0}\right)-\mathrm{C}_{1}{ }^{2}\left(\mathrm{k}_{1} \mathrm{r}_{\mathrm{i}}\right)+\left(\mathrm{k}_{2} \mathrm{a} / \mathrm{k}_{1}\right)^{2}\left[\left(\mathrm{r}_{0}{ }^{2} / \mathrm{r}_{\mathrm{i}}{ }^{2}\right) \mathrm{C}_{2}{ }^{2}\left(\mathrm{k}_{2} \mathrm{r}_{0}\right)-\mathrm{C}_{2}{ }^{2}\left(\mathrm{k}_{2} \mathrm{r}_{\mathrm{i}}\right)\right]\right\} \text { watts } / \mathrm{cm},
\end{gathered}
$$

and the total stored energy on the magnet is:

$$
\begin{gathered}
w_{0}=5 r_{i}^{2}\left(B_{0}^{2} / 8.10^{8}\right) \\
\left\{\left(r_{0}^{2} / r_{i}^{2}\right) C_{1}^{2}\left(k_{1} r_{0}\right)-k_{1}^{2} r_{i}^{2}+a^{2}\left[\left(r_{0}^{2} / r_{i}^{2}\right) C_{2}^{2}\left(k_{2} r_{0}\right)-\left(k_{2}{ }^{2} r_{i}^{2} / 4\right)\right]\right\} \text { joules } / c m,
\end{gathered}
$$

where $\rho$ is the resistivity ( $\rho=1.72 \times 10^{-6}$ for copper) and $\eta$ is the space factor; in the following examples il is assumed that $\eta=0.58$.

More details on the calculations of the magnet are included in Appendix I. 


\section{MAGNET UNITS, RF CAVITIES}

The magnet units consist of a pair of magnet coils enclosed in a cylindrical evacuated tank (Fig. 3). This tank serves also as a radiofrequency cavity where each coil is approximately a quarter wave length at the highest frequency. The gap between the two coils serves as an accelerating gap. In this way, straight sections for pump connections and acceleration are not necessary, thus enabling a smaller circumference length. The tuning of the cavity can be done with rotating condensers up to $1 \%$ of the frequency. The fine tuning $( \pm 1 \%)$ can be accomplished with ferrite coils disposed in series with the rotating condensers. Thus the effect of the low $Q$ of the ferrite is minimized and an overall $Q$ of 1500 is expected for each cavity. Furthermore, by having a plurality of accelerating cavities we minimize the overall rf power requirements. Since the required volts per turn is rather high, in order to keep the amplitude of the phase oscillations within reasonable limits a high harmonic rf order of 30 or more, is required.

The dimensions of the magnet coils and the cavity in an example are:'

$\begin{array}{lrl}\text { Aperture } & 2.5 \mathrm{~cm} \\ \text { Outer coil diameter } & 8 & \mathrm{~cm} \\ \text { Cavity diameter } & 60 & \mathrm{~cm} \\ \text { Cavity length } & 100 & \mathrm{~cm} \\ \text { Field index }\left[\mathrm{n}=\left(\frac{\partial \mathrm{B}}{\partial \mathrm{R}}\right) \frac{\mathrm{R}}{\mathrm{B}}\right] & 450 & \\ \text { Betatron oscillations per turn, " } v " & 8\end{array}$

The acceleration time is assumer as 0.06 seconds. The power source of the magnet can be either motor-generators or a condenser bank. It appears that for such a short acceleration time the cost of a capacitor bank is comparable with the cost of motor-generator units. However, detailed cost estimates are not yet available at this time. In my opinion, though, other conditions being equal, condenser banks are preferable to motor-generators with all their rotating equipment.

In the calculation of the power losses a 60-millisecond linear rise of the magnetic field has been assumed. At the peak value a "flat top" of 10 milliseconds has been included. A decay time of 60 milliseconds has been postulated. Continuous operation of the machine at $60 \mathrm{cps}$ is also possible. In this case, the magnet current varies as

$$
I=I_{0}(1+\cos \omega t) \text {. }
$$


This is accomplished by dc biasing of the magnet. The ac is provided by a capacitor bank. The power loss in this case is rather high. Hence, ac operation must be considered only in a case where very high current is required. It should be noted that a machine built initially for pulsed operation can be converted later to continuous ac operation if higher current is required.

\section{PARTICLE INJECTION, SPACE CHARGE LIMIT}

The number of particles per pulse which can be trapped are limited by the allowed change of the number of betatron oscillations " $v$ " due to the space charge forces in the bunch. We have assumed an (allowable) $\delta v=0.25$ in calculating the number of particles per unit length of the machine. Since the number of trapped particles increases with the injection energy, a higher than usual injection energy can be employed, inasmuch as the linear accelerator cost is not a large part of the overall cost of the machine. For one-turn injection the linear accelerator current becomes too high. Hence, multiturn injection appears more attractive. The most promising way for multiturn in jection, in my opinion, is the molecular injection, namely, to accelerate $\mathrm{H}_{2}{ }^{+}$ and split the molecules upon injection with a mercury jet or otherwise. Of course in this way the particles must be accelerated up to twice the injection energy. However, no other way of multiturn injection appears to me at this time as promising as the molecular injection.

An injection energy of $80 \mathrm{Mev}$ has been postulated. This in turn requires that the $\mathrm{H}_{2}{ }^{+}$ions must be accelerated up to $160 \mathrm{Mev}$. The resulting number of accelerated particles per pulse (for $\delta v=0.25$ ) is $6 \times 10^{12}$. In order to maintain all these particles as they go through the phase transition, it is desirable to avoid any blow-up of the beam at the phase transition. Consequently, a mode of variation of the accelerating voltage was sought which would allow finite amplitude of the phase and momentum oscillations near the transition energy. This investigation was fruitful and it turned out that in a rather simple way one can find solutions for the phase oscillations, thus avoiding the blow-up problem. The required variation of the accelerating voltage $\mathrm{V}$ near the phase transition is given by the equation

$$
\left(\mathrm{V} \cos \phi_{s}\right)-\mathrm{V}_{\mathrm{s}}=\frac{\tau}{\mathrm{T}_{\mathrm{s}}} \mathrm{V}_{\mathrm{i}} \cos \phi_{0}
$$


where $\phi_{S}$ is the instantaneous value of the equilibrium phase; $V_{s}$ is the effect of the space charge of the bunch (which actually at the phase transition energy is negligible); $\tau=t-t_{0} ; t_{0}$ is the time the transition occurs; and $T_{s}$ is a constant. The resulting solution of the phase oscillation is (see Appendix II)

$$
\phi=C_{0} \cos \left(a \tau^{2}+\delta\right)
$$

At the transition point the bunch is exactly at the crest of the wave $\left(\phi_{S}=\pi / 2\right)$, and the accelerating voltage is equal to the threshold voltage-per turn. Beyond the phase transition, the accelerating voltage is gradually increased up to the initial value and the equilibrium phase is restored to the original value but at the other side of the wave. By employing this method it appears that the amplitude of the phase oscillations at the end of the acceleration becomes a small fraction of the betatron oscillations. Since this method is not particular to the proposed accelerator, it can be tried on any of the accelerators now under construction.

\section{ACCELERATOR PARAMETERS}

As an illustrative example, the parameters for a $24-\mathrm{Bev}$ accelerator are listed below.

$\begin{array}{ll}\text { Particle energy (Bev) } & 24\end{array}$

Orbit radius (meters) 16

Aperture (cm) 2.5

Peak value of the magnetic field at the orbit (gauss) 51000

Field index "n" 450

Approximate "v" value 8

Number of magnet units 96

Stored energy in the magnet (megajoules) 3.8

Duration of acceleration (milliseconds). 60

$\begin{array}{ll}\text { Peak rf volts per turn } & 6 \times 10^{6}\end{array}$

Harmonic order (frequency range $32-80 \mathrm{mc}$ ) 32

Injection energy (Mev) ' 80

Number of particles accelerated per pulse $\quad 6 \times 10^{12}$

Final beam cross section $\left(\mathrm{cm}^{2}\right) \quad 0.1$

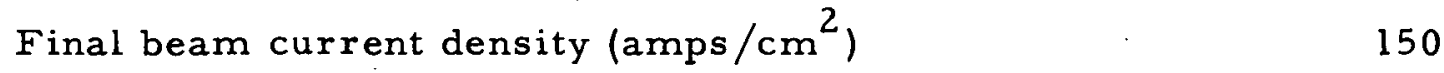


We observe that the final beam current density is high enough to allow synchroclash operation of two machines; namely, the reaction yield for a millibarn.cross section is about 100 per millisecond. It should be noted that particles of a given energy in the laboratory system colliding with a second beam of $25 \%$ of the energy of the first beam would yield in the center of mass system the energy of the first beam. Consequently, the problem to make available the energy of any high-energy machine in the center of mass becomes in the present case quite simple, requiring only an additional expenditure of $25 \%$ of the cost of the machine. Of course much depends on whether or not the number of reactions quoted above are considered satisfactory by the experimental physicists.

A detailed cost estimate for this machine is not available at this time, though preliminary estimates indicate that it is not in excess of a conventional (Brookhaven type), A. G.S. machine.

The same type of machine can be used for continuous ac operation. The cost however would be twice as much, whereas the power requirements are increased by a factor of five approximately. The current, however, becomes 60 microamperes, or more than $10^{14}$ particles per sec. Since such high currents arc not contemplated at this time hy any of the experimental physicists, no further elaboration is warranted at present on the ac operation of the machine.

The aperture of the machine, although it appears small, is actually, relatively speaking, larger than the aperture of the presently-built Brookhaven or CERN machines, because the figure of merit is the product of the field value times the aperture. Hence, the results aresimilar, while the small aperture or the higher field allows much higher beam current, in addition to the important result that the physical size of the machine is smaller. The latter refers, of course, to the circumference of the machine; the cost of the magnet is rather independent (within limits) of the cross section of the magnet.

Finally, it should be emphasized that the above example is cited for purposes of illustration only. The final machine parameters would be determined from more detailed cost estimates and experimental data from the operation of the CERN and Brookhaven machines. 


\section{APPENDIX I}

We shall discuss here in more detail the derivation of the relations for the calculations of the parameters of the coils. As mentioned in Section II, the aperture of the magnet is a cylinder of radius $r_{i}$ and the conductors are located between two cylinders of radius $r_{0}$ and $r_{i}$, respectively. The field components are in the $r, \theta$ (Fig. l) direction only. The current direction is along the $z$-axis, which is parallel to the axis of the cylinders $\left(r_{0}, r_{i}\right)$. The vector potential in the current-free regions can be written for any field with in poles as follows:

$$
\begin{aligned}
\text { region } 0 & <r<r_{i}: \\
A_{z} & =B_{0} r_{i}\left(r^{n} / n r_{i}^{n}\right) \cos (n \theta) ; \\
\text { region } r & >r_{0}: \\
A_{z} & =-c_{0} B_{0} r_{0}\left(r_{0}^{n} / n r^{n}\right) \cos (n \theta),
\end{aligned}
$$

where $c_{0}$ is a dimensionless constant.

Within the coil region we have

$$
\nabla \times(\nabla \times A)=-4 \pi j / 10
$$

where $\mathrm{j}$ is the current density in the coil.

By assuming that

$$
4 \pi j / 10=k^{2} A_{z} \text {, }
$$

the solution of $A_{z}$ is given in Bessel functions of the first and second kind, thus :

$$
A_{z}=-\left(B_{0} / k\right)\left[c_{1} J_{n}(k r)+c_{2} Y_{n}(k r)\right] \cos (n \theta)
$$

or

$$
A_{z}=-\left(B_{0} / k\right) C_{n}(k r) \cos (n \theta) .
$$

The boundary conditions are that the field components are matched at the boundaries and the resulting relations are:

$$
\text { at } \begin{aligned}
r & =r_{i}: \\
& C_{n}^{\prime}\left(k r_{i}\right)=1, \\
& n C_{n}\left(k r_{i}\right) / k r_{i}=1 ;
\end{aligned}
$$




$$
\text { at } \begin{aligned}
\mathbf{r} & =\mathrm{r}_{0}: \\
& \mathrm{C}_{\mathrm{n}}^{\prime}\left(\mathrm{kr} \mathrm{r}_{0}\right)=-\mathrm{c}_{0}, \\
& \mathrm{nC}\left(\mathrm{n}_{\mathrm{n}}\left(\mathrm{kr}_{0}\right) / \mathrm{kr} \mathrm{r}_{0}=\mathrm{c}_{0} .\right.
\end{aligned}
$$

From Eqs. (14) - (15a) and the recurrence formulas, we obtain

$$
\begin{aligned}
& C_{n}^{\prime}\left(k r_{i}\right)-\left[n C_{n}\left(k r_{i}\right) / k r_{i}\right]=-C_{n+1}\left(k r_{i}\right)=0 \\
& C_{n}^{\prime}\left(k r_{0}\right)+\left[n C_{n}\left(k r_{0}\right) / k r_{0}\right]=C_{n-1}\left(k r_{0}\right)=0 .
\end{aligned}
$$

The requirements of Eqs. (16) and (17) result in that

$$
J_{n-1}\left(k r_{0}\right) / Y_{n-1}\left(k r_{0}\right)=J_{n+l}\left(k r_{i}\right) / Y_{n+1}\left(k r_{i}\right) \text {. }
$$

$k$ is determined from Eq. (18) and $c_{0}, c_{1}, c_{2}$ from Eqs. (14) - (16). Finally by substitution in Eq. (12) the current distribution is specified. By constructing the coil with the thus-specified current distribution the desired field can be realized. By assuming the solutions in Bessel functions one has the convenience of existing tabulations, recurrence formulas, etc.

The stored energy in all three regions is obtained from the integral

$$
\mathrm{W}_{0}=\left(10^{-7} / 8 \pi\right) \int_{0,0}^{\infty, 2 \pi} \mathrm{B}^{2} \mathrm{rdr} \mathrm{d} \theta \text { joules } / \mathrm{cm}
$$

and the losses from the integral

$$
\mathrm{w}_{\mathrm{r} 0} \doteq(p / \eta)(10 / 4 \pi)^{2} \mathrm{k}^{4} \int_{\mathbf{r}_{\mathrm{i}}, 0}^{\mathrm{r}_{0}, 2 \pi} \mathrm{A}_{\mathrm{z}}^{2} \mathrm{r} \mathrm{dr} \mathrm{d} \theta \text { watts } / \mathrm{cm}
$$

The actual losses depend on the time variation of the field; in the case where the current varies as $(l+\cos \omega t)$, the actual losses are

$$
\mathrm{w}_{\mathrm{r}}=(3 / 8) \mathrm{w}_{\mathrm{r} 0} \text {. }
$$

Any combination of harmonics can be obtained by adding linearly the corresponding vector potentials.

Analogous solutions can be obtained in axial symmetric field configuration in spherical coordinates; in the spherical case the coil is assumed to be confined between concentric spheres of radius $r_{i}$ and $r_{0}$; then for a $2 n-$ pole field the solution of the vector potential in the coil region is assumed thus:

$$
A_{\phi}=\left(B_{0} / k \sqrt{k r}\right) C_{n+(1 / 2)}(k r)
$$


with the condition that:

$$
\begin{aligned}
& C_{n+(3 / 2)}\left(k r_{i}\right)=0
\end{aligned}
$$

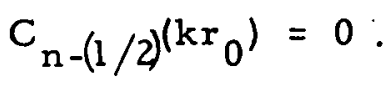

The other constants are determined by the same procedure as in the cylindrical case.

In the cylindrical case we observe from Eqs. (20) and (21) that the power loss is independent of the scaling factor, whereas the stored energy is proportional to the square of the radius $r_{i}$.

The actual coil construction presents some practical difficulties. Since many conductors must be connected in parallel, it is required that the emf is the.same in each group of parallel conductors; otherwise the current distribution will be disturbed. The emf is in the $z$-direction; namely,

$$
E_{\mathbf{z}}=-\frac{1}{c} \dot{A}_{\mathbf{z}}
$$

We observe that according to Eq. (24) the emf is constant along surfaces of constant vector potential. This in turn means that the emf is constant on surfaces whose trace on a plane $z=$ constant are magnetic lines. Hence groups of conductors connected in parallel must be placed on such surfaces. If $\mathrm{N}$ is the (total) number of conductor groups desired to be connected in series, we shall divide the coil area in $\mathrm{N}$ zones where in each zone

$$
\int \mathrm{jr} d r d \theta=I_{0} / \mathrm{N}
$$

where $I_{0}$ is the total current in the coil (in the case where all the conductors were' connected in parallel). After the zones have been determined, the conductors are placed in the center of each zone. 


\section{APPENDIX II}

The well-known equation for the phase oscillations is

$$
\begin{aligned}
& \frac{d}{d t}\left(\frac{\gamma}{\Gamma} \cdot \frac{d \phi}{d t}\right)+\omega_{0}^{2} \frac{h v_{i} \cos \phi_{0}}{2 \pi V_{0}} \phi=0, \\
& \text { where } \quad \Gamma=\left(\frac{1}{\gamma^{2}}-\frac{1}{v^{2}}\right) \\
& \gamma=E / M c^{2} \text { (the relativistic mass ratio) } \\
& v=\text { the number of betatron oscillations per turn } \\
& \omega_{0}=c / R \text { the Larmor frequency of a particle with velocity } c \\
& \mathrm{~h}=\text { the harmonic order } \\
& v_{0}=M_{0} c^{2} / e \\
& \phi=\text { the phase angle in respect to the equilibrium phase } \\
& \phi_{0}=\text { the equilibrium phase } \\
& \mathrm{V}_{\mathrm{i}}=\text { the applied } \mathrm{rf} \text { voltage per turn. }
\end{aligned}
$$

The phase transition occurs when

$$
\gamma \equiv \gamma_{0}=\nu \text {. }
$$

The adiabatic approximate solution of Eq. (26) is

$$
\phi=\operatorname{const}(\Gamma / \gamma)^{1 / 4} \cdot \cos \left(\int \Omega \mathrm{dt}+\delta\right),
$$

where

$$
\Omega=\left(\frac{\mathrm{v}_{\mathrm{i}} \cdot \cos \phi_{0} \Gamma}{2 \pi \mathrm{V}_{0} \gamma}\right)^{1 / 2} .
$$

Near the phase transition the approximation is not valid and it is customary to assume that $\Gamma$ is varying linearly. with time. Then the solutions admit ted are Bessel and Neumann functions of order $2 / 3.1$ In the present case this solution.was not considered satisfactory and a new one was sought in order to avoid beam blow-up during the phase transition. I assumed that near the phase transition the equilibrium phase is shifted with time according to the equation

$$
\left(\mathrm{V} \cos \phi_{s}\right)-\mathrm{V}_{\mathrm{s}}=\frac{\tau}{\mathrm{T}_{\mathrm{s}}}\left(\mathrm{V}_{\mathrm{i}} \cdot \cos \phi_{0}\right)
$$

where $V_{S}$ results from the space charge of the bunch (this quantity is huwever negligible at the phase transition and it can be deleted from the equation); 
$\phi_{s}, \mathrm{~V}$ are the instantaneous values of the equilibrium phase and applied accelerating voltage, respectively; $\tau=t-t_{0} ; t_{0}$ is the time the transition occurs, and $T_{s}$ is a constant with dimensions of time. This quantity must be larger than the transition mistiming due to momentum spread.

The quantity $\Gamma$ varies with time as:

$$
\Gamma=\frac{1}{\gamma^{2}}-\frac{1}{v^{2}}=-\left(\tau / \gamma_{0}^{2} T_{0}\right) \text {, }
$$

where

$$
\mathrm{T}_{0}=\left(\gamma^{3} / 2 \dot{\gamma} \gamma_{0}^{2}\right) \approx \cdot\left(\gamma_{0} / 2 \dot{\gamma}\right)
$$

and

$$
\dot{\gamma}^{\cdot}=\dot{\mathrm{E}} / \mathrm{M}_{0} \mathrm{c}^{2}
$$

Upon substitution in Eq. (26) we obtain

$$
\ddot{\phi}-\frac{\dot{\phi}}{\tau}+\omega_{1}^{2}\left(\frac{\tau^{2}}{\mathrm{~T}_{0} \mathrm{~T}_{\mathrm{s}}}\right) \phi=0,
$$

where

$$
\omega_{1}=\omega_{0}\left(h v \cos \phi_{0} / 2 \pi v_{0} \gamma_{0}^{3}\right)^{1 / 2} \text {. }
$$

Equation (33) admits the solution

$$
\phi=\frac{\tau}{T_{s}}\left[c_{1} J_{1 / 2}\left(a \tau^{2}\right)+c_{2} Y_{1 / 2}\left(a \tau^{2}\right)\right],
$$

where

$$
a=\frac{\omega_{1}}{2 T_{s}^{1 / 2} T_{0}^{1 / 2}}
$$

Substituting the Bessel and Neumann functions by their asymptotic expansions we obtain:

$$
\phi:=c_{1} \cos \left(a \tau^{2}\right)+c_{2} \sin \left(a \tau^{2}\right)
$$

or

$$
\phi=c_{0} \cos \left(a \tau^{2}+\delta\right)
$$

We observe that Eq. (37) is the desired solution. As long as the equilibrium phase varies as prescribed by Eq. (27), the amplitude of the phase oscillations remains constant; thus blow - up of either phase or momentum oscillations is avoided.

Equation (31) indicates that $\dot{\gamma}$ must remain constant during the time the phase shifts. Consequently the applied voltage times the sine of the

$$
895.915
$$


equilibrium phase must remain constant during the same time interval; namely,

$$
\mathrm{V} \sin \phi_{s}=\mathrm{V}_{\mathrm{i}} \cdot \sin \phi_{0}
$$

Equations (29) and (38) yield

$$
\begin{aligned}
\cot \phi_{S} & =\tau / T_{s} \\
\sin \phi_{s} & =\left[\frac{1}{1+\left(\frac{\tau}{T_{s}}\right)^{2}}\right]^{1 / 2} \\
V & =\left(V_{i} \sin \phi_{0}\right) \sqrt{1+\left(\frac{\tau}{T_{s}}\right)^{2}} .
\end{aligned}
$$

At ' $t=t_{0}$, at the phase transition point, the equilibrium phase is $\pi / 2$, the applied voltage becomes equal to the threshold voltage per turn and the bunch rides at the crest of the accelerating wave.

The amplitude of the momentum oscillations and the corresponding radial oscillations are

$$
\begin{aligned}
& \frac{\delta p}{p}=c_{0}\left[\frac{\left(v_{i} \cdot \cos \phi_{0}\right) T_{0} \cdot \gamma_{0}}{\left(2 \pi h v_{0} T_{s}\right)}\right]^{1 / 2} \\
& \frac{\delta R}{R}=\left(\frac{\delta p}{p}\right) / \gamma_{0}^{2} .
\end{aligned}
$$

The constants $c_{0}, \delta_{0}$ are determined from Eq. (28) at $\tau=-T_{s}$, i. e, at the time where the applied voltage starts to vary in accordance with Eq. (41). Due to the momentum spread all of the particles are not going through the phase transition simultaneously. Consequently, due to this mistiming error as well as nonlinear effects, both the amplitude of the phase and momentum oscillations are expected to be somewhat higher than indicated from the above calculations. 


\section{ACKNOW LEDGMENTS}

The author is indebted to Drs. Lloyd Smith and David Judd, Lawrence Radiation Laboratory, University of California, Berkeley, and Dr. Kenneth Simon, Midwestern Universities Research Association, for fruitful discus sions during the preparation of this study.

\section{REFERENCE}

1. E. D. Courant and H.S. Snyder, Annals of Physics, Vol. 3 (1958) pp. 40. 


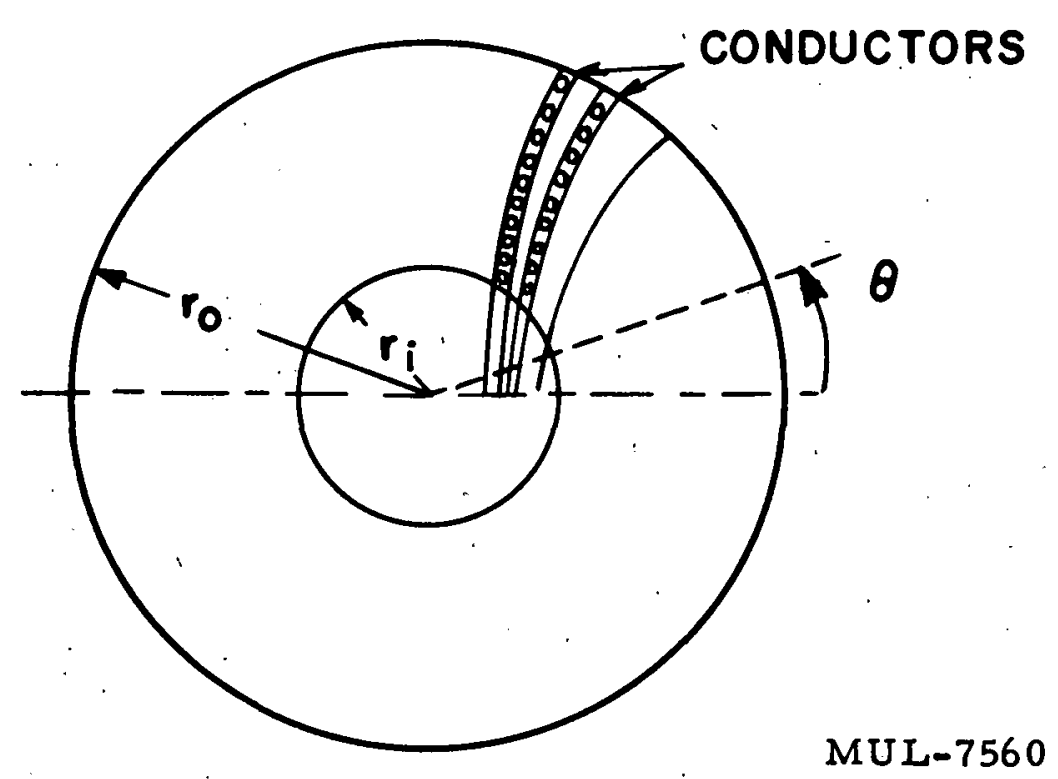

Figure 1.

8ิ3 


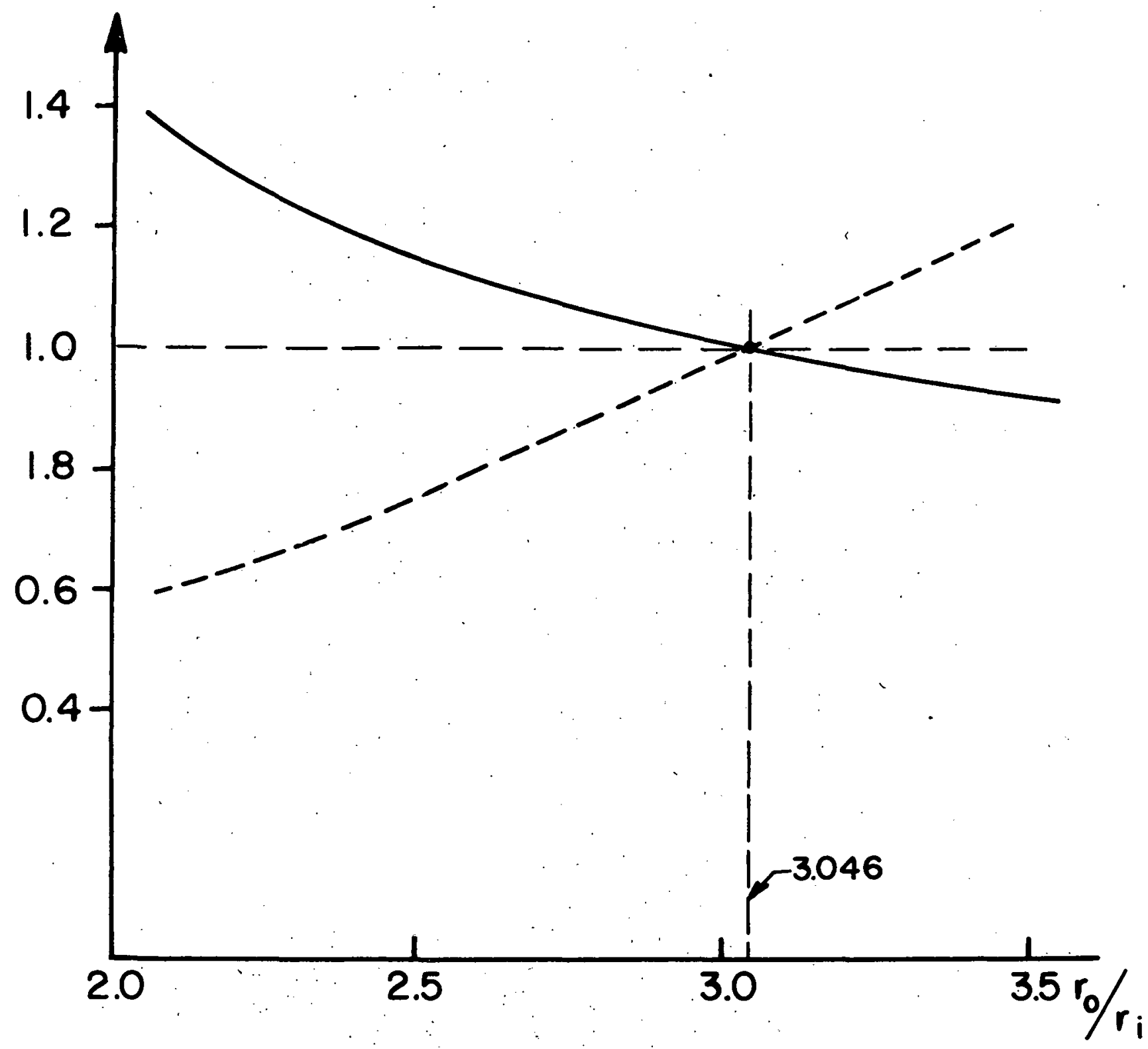

- - Stored energy

Power loss

Figure 2. 


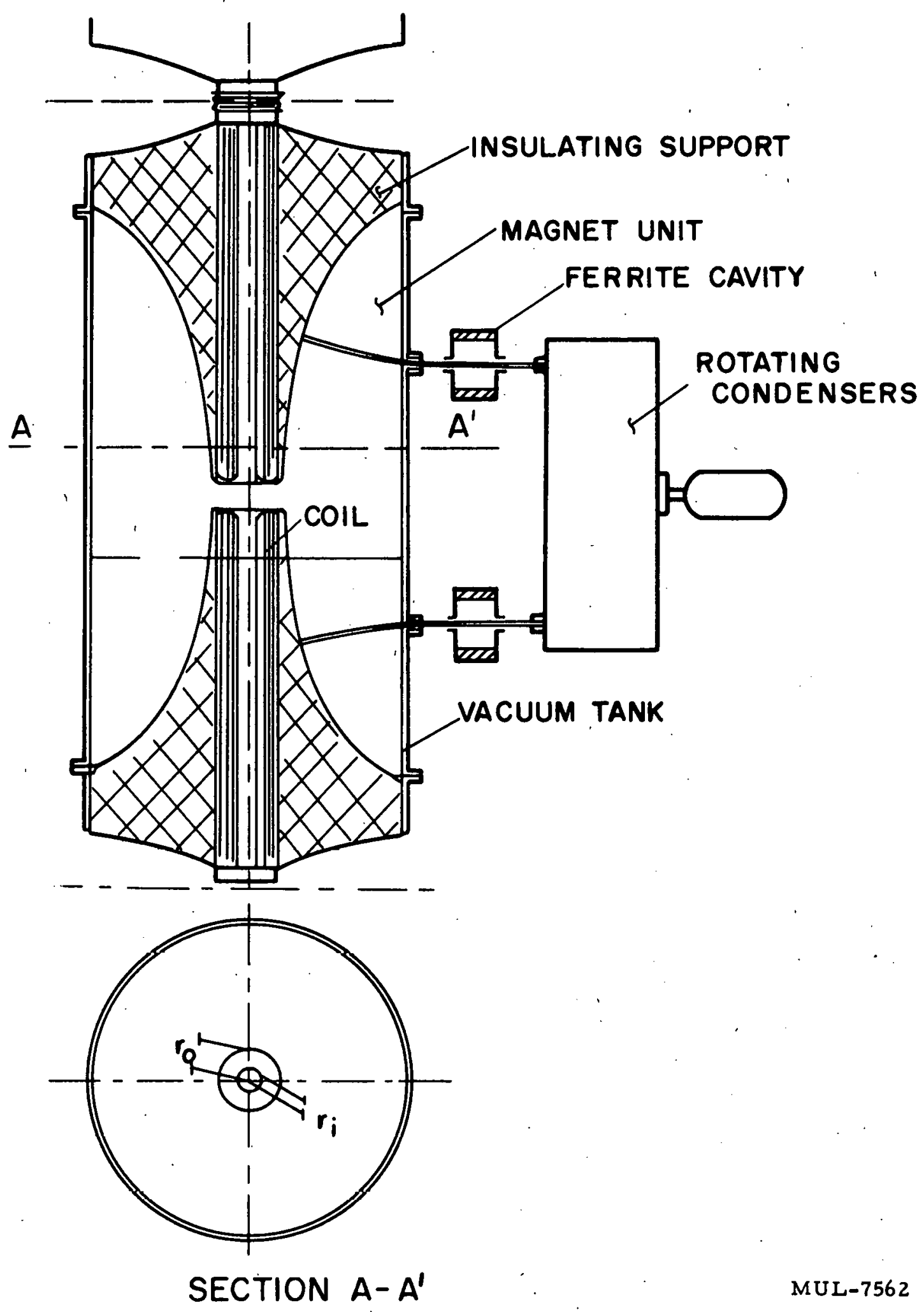

Figure 3. 